\title{
QUALIDADE DOS DADOS SOBRE ÓBITOS NEONATAIS PRECOCES
}

Linda Délia Carvalho de Oliveira Pedrosa*, Silvia Wanick Sarinho, Ricardo Arraes de Alencar Ximenes, Manoelina R. Ordonha

Trabalho realizado no Centro de Ciências da Saúde da Universidade Federal de Pernambuco

\section{*Correspondência}

Condomínio Aldebaran Beta

Quadra C Lote 2 Jardim

Petrópolis Maceió, Alagoas

CEP $57080-900$

Fone (82) 3358-5294

cel: $(82) 9983-8093$

lindapedrosa@uol.com.br

\section{RESUMO}

Objetivo. Verificar a qualidade dos dados oficiais de óbitos neonatais em Maceió, Alagoas.

МéтоDo. Estudo descritivo dos óbitos neonatais precoces hospitalares ocorridos entre primeiro de janeiro de 2001 e 31 de dezembro de 2002, comparando o preenchimento da Declaração de Óbito (DO) e do Sistema de Informação sobre Mortalidade (SIM) com um formulário padronizado, preenchido com dados dos prontuários de mães-neonatos. Estudouse a freqüência e omissão de registro na DO e no SIM das variáveis: tipo de óbito, endereço, idade materna, sexo, peso ao nascer, tipo de parto, idade no óbito e idade gestacional, verificando a confiabilidade do SIM por concordância simples, sensibilidade e Indicador Kappa.

Resultados. O SIM registrou 45 I óbitos, dos quais foram excluídos 50. Houve 44, $1 \%$ de omissão da variável idade materna no SIM. Nos prontuários, foram resgatadas $85,7 \%$ a $100 \%$ das variáveis no preenchidas na DO. A concordância foi boa entre DO e prontuário para tipo de parto, peso ao nascer e idade. As variáveis peso ao nascer e idade materna apresentaram a menor concordância entre pronturios e o SIM, que apresentou sensibilidade de 69,2\% para peso ao nascer e de 36,3\% para idade materna, demonstrando pouca capacidade de informar corretamente variáveis importantes para geração de indicadores da saúde perinatal.

Conclusão. A qualidade precária da DO decorrente do seu preenchimento incompleto torna o SIM inadequado, mesmo abrangendo $100 \%$ dos óbitos neonatais da cidade. A ineficiência do sistema aumenta, devido a não correção das falhas encontradas e a não digitação de todas as informações disponíveis por parte dos técnicos do SIM.

Unitermos: Mortalidade neonatal. Atestados de óbito. Sistemas de informação. Registros médicos. Registros de mortalidade. Estudo de validação.

\section{INTRODUÇÃO}

O médico é responsável pelo preenchimento da Declaração de Óbito (DO)', documento que, além de permitir o sepultamento, alimenta, no âmbito nacional, o Sistema de Informação sobre Mortalidade $(\mathrm{SIM})^{2}$

Apesar de ser o mais antigo dos Sistemas de Informações em Saúde (SIS) nacionais, ainda é difícil analisar a mortalidade neonatal a partir dos dados do SIM, por (todos) estes serem, em muitos casos, insuficientes e conflitantes e que, aliados cobertura heterogênea do Sistema, podem comprometer sua validade na maioria dos municípios brasileiros ${ }^{3,4}$.

No decorrer dos anos, melhorias foram introduzidas no Sistema visando melhor adequação e agilidade: a descentralização e, na dependência do município, outras atividades como o controle do preenchimento da DO, correção de endereços e busca de esclarecimentos sobre causas mencionadas de modo incompleto. A padronização de coleta e fluxo dos documentos, capacitação dos técnicos, o uso do Programa Seletor de Causa Básica, além da diminuição do registro de óbitos de causa mal definida, permitiram a maior agilidade na conclusão e consolidação dos bancos de dados. A cobertura do SIM, entretanto, em 2002 se situava em média $83,15 \%$, menor no Nordeste $(68,70 \%)$ e maior na região Sul $(90,58 \%)^{5-7}$.
A cobertura e confiabilidade do SIM são características essenciais que the conferem credibilidade, mas que ficam comprometidas pela subnotificação de eventos, preenchimento incorreto e incompleto da DO, presença de discordâncias e quando há elevado percentual de variáveis ignoradas. Incompletas, suas informações podem distorcer os indicadores gerados, a ponto de transmitir uma falsa idéia de adequação das condições de saúde locais². As variáveis da DO, utilizadas na geração de indicadores, são classificadas para efeito de crítica e correção pelo Sistema em indispensáveis: tipo e data do óbito; essenciais: sexo, idade e município de residência; e secundárias: todas as demais, inclusive as de preenchimento exclusivo para óbitos fetais e em menores de um ano ${ }^{4}$. A omissão dos antecedentes obstétricos em óbitos neonatais pode ocasionar sub-estimativa dos eventos ocorridos na gestação e parto, que culminam com as causas maternas, na maioria, evitáveis ${ }^{3}$.

Alagoas detém o maior índice de mortalidade infantil do país (com taxa de 55,57/1000 nascidos vivos em 2001, e de 52,64 / 1000 nascidos vivos em $2002^{8}$ ) e não dispõe de estudos que avaliem a qualidade das informações oficiais de óbitos neonatais.

O objetivo do estudo é avaliar a qualidade do Sistema de Informações sobre Mortalidade, por meio do estudo das variáveis da Declaração de Óbito e de dados de correspondente transcrição dessas variáveis em campos dos registros do sistema. 
Pedrosa LDCO et al.

\section{Métodos}

Estudo descritivo exploratório, para verificar a qualidade do SIM em residentes de Maceió, capital de Alagoas, situada na região Nordeste do país, utilizando dados relativos aos óbitos neonatais precoces hospitalares ocorridos nesta cidade entre primeiro de janeiro de 2001 e 31 de dezembro de 2002.

A Secretaria Municipal de Saúde de Maceió (SMSM) disponibilizou cópias das DOs preenchidas pelos médicos e acesso aos registros do SIM digitados por técnicos. Os prontuários foram localizados nos arquivos hospitalares. A população do estudo foi composta por 453 óbitos não fetais, ocorridos em menores de até seis dias de vida completos, cujos endereços foram validados através do código de endereçamento postal. As DOs fetais também foram revisadas para detectar erros de classificação. Para detectar possíveis DOs sem registro na SMSM, foi realizada a busca ativa dos registros dos óbitos nos Cartórios de Registro Civil de Maceió.

No presente estudo, os dados da DO e as correspondentes transcrições destes nos registros das respectivas DOs no SIM, de um lado, foram comparados com dados recolhidos em formulário padronizado, a partir dos registros de prontuários médicos.

Foram analisadas as variáveis: tipo de óbito, endereço, idade materna, sexo, peso ao nascer, tipo de parto, idade no óbito, idade gestacional e assistência médica. As variáveis qualitativas foram categorizadas em: tipo de óbito (fetal e não-fetal); tipo de parto (operatório e não-operatório: natural e fórceps); sexo (feminino, masculino e indeterminado); idade gestacional ( $<22$ semanas, 2227 semanas, 28-31 semanas, 32-36 semanas, 37-4I semanas, 42 e mais semanas, ignorado); assistência médica (sim e não). As variáveis quantitativas foram categorizadas para permitir a análise de concordância pelo Indicador Kappa. Foram utilizados os seguintes pontos de corte: Idade na data do óbito: $\leq$ I dia e > I dia de vida; peso ao nascer: < 2500 g e $\geq 2500$ g; Idade materna: $\leq 19$ anos (adolescentes) e $>19$ anos.

A qualidade dos documentos foi analisada pela freqüência do preenchimento das variáveis selecionadas e o grau de confiabilidade do SIM, pela análise de concordância entre os prontuários DO e SIM, análise ajustada para todas as variáveis quantitativas e qualitativas e por meio do Indicador Kappa e seus intervalos de confiança (considerando: $<0,00$ - ruim; 0,00 - 0,20 - fraca; 0,21 - 0,40 - sofrível; 0,41 - 0,60 - regular; 0,61 - 0,80 - boa; 0,81 - 0,99 - ótima e 1,00 perfeita) 9 . A validade do SIM foi verificada por meio de medidas de sensibilidade (ou taxa de detecção), considerando-se o formulário da pesquisa como padrão-ouro, comparando suas informações com as constantes nas DOs e no SIM. A sensibilidade do Sistema e da DO foi calculada como a proporção entre as informações corretas nos prontuários e também nas DOs ou SIM (verdadeiros-positivos), divididas pela soma destes verdadeiros-positivos e os falsos-negativos (informações desconhecidas no formulário por serem inexistentes nos prontuários, mas preenchidas na DO ou no Sistema). O programa Epi Info 6.04 foi utilizado para digitação em dupla entrada, validação e análises estatísticas dos dados.

Foram estudados todos os óbitos neonatais hospitalares ocorridos nesta capital, no período determinado, de mães residentes em
Maceió. O quantitativo de óbitos verificados no estudo está contemplado no montante calculado pela fórmula para estudos de validação $^{10}$, com power de $80 \%$ e erro de $5 \%$, baseada em valores de sensibilidade da DO em detectar as causas neonatais de óbito de 83,3\%", resultando em $n$ de 334 óbitos.

A pesquisa obteve aprovação do Comitê de Ética em Pesquisa da Universidade Federal de Alagoas em 25 de novembro de 2003, processo no. 009571/2003-10.

\section{Resultados}

Entre 2001 e 2002, o SIM registrou 45I óbitos neonatais precoces hospitalares de residentes em Maceió, dos quais foram excluídos 50: um adulto, 12 óbitos fetais, um óbito domiciliar, duas DOs duplicadas, sete óbitos de maiores de sete dias de vida e 27 óbitos de filhos com mães residentes em outros municípios, após averiguação dos dados. A exclusão destes óbitos reduziu em I I, I\% o total de óbitos atribuídos àquela faixa etária entre os filhos de mães residentes no Município de Maceió no período estudado. As perdas do estudo corresponderam a $14,7 \%$ (59/40I) dos óbitos. Suas características foram analisadas e não apresentaram diferença estatisticamente significante em relação aos óbitos estudados. Foi encontrado um óbito neonatal precoce entre as DOs fetais e incluído na pesquisa. Apenas 4I,4\% (166/40I) dos óbitos incluídos do SIM foram registrados nos cartórios de registro civil.

Foram revisados $401 / 45$ I registros no SIM, 396/45I DOs e $343 / 45$ I prontuários. As características da população da pesquisa foram definidas a partir dos 343 prontuários estudados, dos quais 22,7\% (78/343) tinham peso ao nascer $\geq 2500$ g; 55,4\% (190/ $343)$ eram do sexo masculino; $53,6 \%$ (184/343) faleceram nas primeiras 24 horas de vida; 65\% (222/343) nasceram de parto não operatório; 18,6\% (64/343) eram a termo, com idade gestacional $\geq 37$ semanas. As mães tinham idade $\leq 19$ anos em 31,5\% (108/ 343) dos óbitos.

Em 22\% (87/396) das DOs, no foram assinalados pelo médico o tipo de óbito (fetal/não fetal); em 3,5\% (14/396) foram assinalados o tipo fetal e ambos em $0,5 \%$ dos óbitos (02/396). 0 endereço incompleto em 5,8\% (23/396) DOs impossibilitava determinar o município de residência, obrigando a busca dos prontuários.

A caligrafia pouco legível do médico dificultou o entendimento pelos técnicos da SMSM dos endereços, sendo também encontrados nomes maternos incompletos ou errados. Endereços ausentes, errados e não corrigidos, em algumas DOs, resultaram na inclusão de 27/45। DOs de outros municípios. Em 4,3\% (I7/ 396) das DOs não havia sido assinalada pelo médico a variável "sexo", porém este dados foi informados todas as vezes no SIM. Em duas DOs com sexo "indefinido", devido a malformações congênitas, o sexo "masculino" foi assinalado no SIM. A variável menos preenchida pelos médicos foi "idade materna", chegando a 44,2\% (175/396) de omissão, quando apenas um caso não dispunha desta informação no prontuário. Apenas duas crianças gêmeas imperfeitas (peso ao nascer aproximado 5000 g), não tiveram peso ao nascer individual determinado nos prontuários, 
sendo esta variável omitida em 15,2\% (61/396) das DOs. A variável assistência médica esteve ausente em 68,4\% (200I) e em 62,2\% (2002) dos óbitos. As variáveis em branco na DO foram resgatadas nos prontuários em 85,7\% a 100\% dos óbitos (Tabela I).

No SIM não foram digitados dados fundamentais como o peso ao nascer, em 60,4\% (242/40I) dos óbitos, omitindo ao sistema 44,1\% (147/335) dos assinalados pelos médicos nas DOs, resultando em elevado número de dados "missing". As variáveis "tipo de óbito" e "sexo" foram todas informadas.

A concordância entre o prontuário e a DO (Tabela 2) foi considerada boa em tipo de parto, peso ao nascer e idade.

Entre os prontuários, em que se admitem estar os verdadeiros dados do paciente, e o SIM (Tabela 3), a variável peso ao nascer obteve menor concordância (42,6\%), Kappa de 0,19; seguida da idade materna, concordante em 54,6\% e Kappa de 0,34. O SIM apresentou uma sensibilidade de $69,2 \%$ na variável peso ao nascer e de $36,3 \%$ em idade materna.

\section{Discussão}

A DO não pode ser vista apenas como instrumento legal, pois seus dados geram indicadores que subsidiam não apenas os programas locais de atenção à saúde, como estatísticas no âmbito local, nacional e mundial. Permitem sua utilização em pesquisas internacionais, como as realizadas pelas redes de cooperação em pesquisas, a exemplo da Rede Vermont-Oxford (VON), Rede Neonatal do Instituto de Saúde Infantil (NICHD) e a Rede Brasileira de Pesquisas Neonatais (RBPN) ${ }^{12}$

Esta pesquisa detectou a inadequação do SIM em Maceió. Nos registros de óbitos fetais também foram encontrados erros de classificação, possivelmente pela dificuldade e até pelo desconhecimento das definições de nascido vivo e perdas fetais, fato já observado em outros estudos ${ }^{3,4}$. A ausência de crítica de inconsistências em relação à causa básica e à idade também geraram erros que a averiguação rotineira no sistema é capaz de detectar. Por outro lado, foram estudados apenas os óbitos de residentes em Maceió, ocorridos nesta capital. Ao se verificar este critério na seleção dos óbitos para o estudo, observou-se também a não validação de endereços e a aceitação de DO sem endereço firmado. Dos 27 óbitos excluídos por endereço inconsistente com o prontuário, é possível que alguns representem invasão de óbitos de outros municípios, pois Maceió é uma cidade com melhores recursos de atenção à saúde.

A subnotificação, outro fator limitante do SIM por reduzir a quantidade de dados disponíveis, foi observada no estudo de Santa Helena et al. ${ }^{13}$, em 7,3\% de óbitos não fetais e em $11,1 \%$ fetais, e também relatada em estudos internacionais de países como a Índia, onde a publicação de estatísticas oficiais é irregular e não sistemática $^{14}$. Os percentuais elevados de sub-registro encontrados no presente estudo provavelmente se devem ao desconhecimento ou desinteresse dos responsáveis pelos menores em proceder este registro, por se tratarem de óbitos em menores de seis dias, quando não há nenhuma aparente necessidade de registrá-los, além do trabalho de ir ao cartório. Também é importante na redução de

\begin{tabular}{|c|c|c|c|c|}
\hline $\begin{array}{r}\text { Tabela I - Distr } \\
\text { óbito, das variáv } \\
\text { parto e idade ge } \\
\text { em óbitos }\end{array}$ & $\begin{array}{l}\text { freqüênc } \\
\text { o nascer, } \\
\text { e resgate } \\
\text { hospitalar }\end{array}$ & $\begin{array}{l}\text { oreencl } \\
\text { idaden } \\
\text { ariáveis } \\
\text { ecoces, }\end{array}$ & $\begin{array}{l}\text { imento, nas declar } \\
\text { óbito, idade da m } \\
\text { em branco nos prc } \\
\text { em Maceió, 200I-2 }\end{array}$ & $\begin{array}{l}\text { es de } \\
\text { po de } \\
\text { ários, }\end{array}$ \\
\hline Variável & $\begin{array}{r}\text { Val } \\
\text { em bra }\end{array}$ & DO & $\begin{array}{r}\text { Variáve } \\
\text { resgatadas em p }\end{array}$ & uários \\
\hline & $\mathrm{N}^{\circ}$ & $\%$ & $\mathrm{~N}^{\circ}$ & $\%$ \\
\hline $\begin{array}{l}\text { Sexo } \\
\text { Idade no óbito } \\
\text { Peso ao nascer } \\
\text { Idade materna } \\
\text { Tipo de parto } \\
\text { Idade gestacional }\end{array}$ & $\begin{array}{l}14 / 337 \\
28 / 337 \\
46 / 337 \\
135 / 337 \\
50 / 337 \\
61 / 337\end{array}$ & $\begin{array}{l}4,2 \\
8,3 \\
13,7 \\
40,0 \\
14,9 \\
18,1\end{array}$ & $\begin{array}{c}12 / 144^{*} \\
24 / 28 * \\
44 / 46 * \\
134 / 135 * \\
50 / 50 * \\
41 /\left.6\right|^{*}\end{array}$ & $\begin{array}{l}85,7 \\
85,7 \\
95,6 \\
99,2 \\
100,0 \\
67,2\end{array}$ \\
\hline
\end{tabular}

* no numerador total de informação resgatada no prontuário e no denominador total de informação em branco na DO para cada variável.

subnotificações a exigência do registro pelos cemitérios, para proceder o sepultamento. A pesquisa detectou uma DO no arquivo hospitalar, mas o referido óbito foi não informado à SMSM, donde se conclui o seu enterro clandestino.

A qualidade da DO preenchida pelos médicos mostrou-se inadequada, uma vez que o percentual elevado de omissão de uma variável indispensável ${ }^{5}$ como tipo de óbito, aponta para o desconhecimento dos médicos da sua obrigatoriedade e da distinção de óbito fetal e nascido vivo e impossibilita a entrada do registro no sistema.

Os estudos no Brasil sobre a qualidade do preenchimento das DOs e, portanto, de estudo da qualidade do SIM sob a ótica da disponibilidade de informações, datam de 1970 a 199013,16-19. No entanto, pesquisas atuais ${ }^{5}$ no país não demonstram melhora significativa nestes percentuais. Nos países desenvolvidos, o mau preenchimento das DOs é verificado em maior magnitude em relação à causa de óbito, para onde a maior parte das pesquisas converge ${ }^{19-25}$. Lomuto ${ }^{26}$, na Argentina, relata o registro deficiente da idade gestacional comprometendo a análise do percentual de baixo peso e prematuridade. Hetoghe et al. ${ }^{27}$, na Bélgica, encontraram concordância no peso ao nascer em $69 \%$ dos óbitos estudados, com maior sub-registro de informações em neonatos de baixo peso. Neste estudo, foi observado que Maceió apresenta, atualmente, padrão de qualidade do preenchimento da DO semelhante ao encontrado em regiões mais desenvolvidas do país (Sul e Sudeste) há algumas décadas.

Maceió, que possui população de 884.320 habitantes é o $18^{\circ}$ município brasileiro em população, sendo que existem 5548 municípios brasileiros com população inferior ${ }^{28}$. As informações coletadas de forma inadequada, em municípios com características semelhantes, distorcem e se refletem nos indicadores do Brasil como um todo. Portanto, a melhoria do SIM em Maceió é necessária.

O estudo da consistência do SIM em relação às DOs sugere ausência de crítica na digitação e de verificação da consistência dos dados pela SMSM, principalmente em 2001, quando importantes dados não foram transmitidas ao SIM. Aqui estão incluídos os dados 
PedRosa LDCO et al.

\begin{tabular}{|c|c|c|c|c|c|}
\hline Variáveis & $\begin{array}{l}\text { Concordância simples } \\
\text { (\%) }\end{array}$ & $\begin{array}{l}\text { Sensibilidade } \\
\text { (S) }\end{array}$ & IC. $595 \%$ & $\begin{array}{c}\text { Kappa } \\
\text { (K) }\end{array}$ & IC-K95\% \\
\hline $\begin{array}{l}\text { Sexo } \\
\text { Tipo de parto } \\
\text { Peso ao nascer } \\
\text { Idade no óbito } \\
\text { Idade materna } \\
\text { Idade gestacional }\end{array}$ & $\begin{array}{l}93,0 \\
84,2 \\
84,3 \\
84,0 \\
58,7 \\
79,2\end{array}$ & $\begin{array}{l}92,7 \\
89,8 \\
90,2 \\
84,2 \\
40,2 \\
91,6\end{array}$ & $\begin{array}{l}87,9-95,8 \\
84,6-93,4 \\
85,7-93,3 \\
78,1-88,9 \\
32,9-48,1 \\
87,0-94,7\end{array}$ & $\begin{array}{l}0,87 \\
0,71 \\
063 \\
0,71 \\
0,38 \\
0,58\end{array}$ & $\begin{array}{l}0,77-0,97 \\
0,62-0,78 \\
0,55-0,71 \\
0,61-0,80 \\
0,32-0,43 \\
0,50-0,66\end{array}$ \\
\hline \multicolumn{6}{|c|}{ Tabela 3 - Concordância para variáveis selecionadas entre prontuários e o SIM, em óbitos neonatais precoces, em Maceió, 200I-2002 } \\
\hline Variáveis & $\begin{array}{l}\text { Concordância simples } \\
(\%)\end{array}$ & $\begin{array}{l}\text { Sensibilidade } \\
\text { (S) }\end{array}$ & IC-S95\% & $\begin{array}{l}\text { Kappa } \\
\text { (K) }\end{array}$ & IC-K95\% \\
\hline $\begin{array}{l}\text { Sexo } \\
\text { Tipo de parto } \\
\text { Peso ao nascer } \\
\text { Idade no óbito } \\
\text { Idade materna } \\
\text { Idade gestacional }\end{array}$ & $\begin{array}{l}94,0 \\
80,7 \\
42,6 \\
89,0 \\
54,6 \\
80,6\end{array}$ & $\begin{array}{l}92,1 \\
86,3 \\
69,2 \\
85,2 \\
36,3 \\
90,9\end{array}$ & $\begin{array}{l}87,2-95,3 \\
80,8-90,5 \\
61,6-76,0 \\
79,5-89,6 \\
29,2-44,0 \\
86,1-94,2\end{array}$ & $\begin{array}{l}0,88 \\
0,65 \\
0,19 \\
0,78 \\
0,34 \\
0,55\end{array}$ & $\begin{array}{l}0,78-0,98 \\
0,57-0,73 \\
0,15-0,23 \\
0,68-0,88 \\
0,30-0,38 \\
0,47-0,63\end{array}$ \\
\hline
\end{tabular}

referentes à parte $\mathrm{V}$, exclusiva para óbitos fetais e de menores de um ano, mesmo quando presentes na DO. A determinação da idade, predominantemente pela diferença entre as datas de nascimento e óbito, permitiu a inclusão de $06 / 451(1,3 \%)$ óbitos neonatais tardios.

É importante observar que o preenchimento das DOs pelos médicos foi um procedimento adequado para a maioria das variáveis estudadas, uma vez que a sensibilidade da DO foi baixa apenas para a variável idade materna. Ao transcrever no SIM as informações da DO, os digitadores omitiram uma série de dados, fazendo com que este banco de dados passasse a não mais refletir as informações reais dos neonatos, que são aquelas constantes nos prontuários médicos. Estatisticamente, esta observação ficou expressa na queda da concordância simples, concordância ajustada pelo Kappa, sensibilidade do sistema em relação ao prontuário, patente em todas variáveis, exceto sexo e idade gestacional, e principalmente em peso ao nascer e idade materna.

Estes achados demonstram quanto o SIM pouco reflete da realidade sobre os óbitos ocorridos em Maceió, em relação aos dados que aferem a qualidade da assistência prestada à gestante e ao concepto, e norteiam ações preventivas. A baixa sensibilidade do sistema demonstra a sua restrita capacidade em detectar e informar, corretamente, reconhecidas variáveis preditoras de risco para óbito, como peso ao nascer e idade materna. Desta forma, o SIM deixa de exercer parte de suas funções como Sistema de Informação em Saúde (SIS). A maior sensibilidade do sistema em detectar informações sobre idade gestacional, embute também certo grau de imprecisão, pois na DO a idade gestacional é aferida em faixas de semanas.

Em Recife, Vanderlei et al. ${ }^{20}$ ressaltam o ganho de apenas $50 \%$ dos dados nos prontuários. Apesar desta e de outras referências desalentadoras quanto ao preenchimento dos prontuários, a exemplo de Coutinho ${ }^{4}$, Serafim ${ }^{29}$ e Campos $^{30}$, para quem o mau preenchimento dos formulários e prontuários reflete a má qualidade da assistência prestada. No presente estudo, a quantidade de dados resgatados nos prontuários foi elevada provavelmente por terem ocorrido, em sua maioria, em unidades de instituições de ensino, permitindo concluir que possivelmente não foi desconhecimento da informação que determinou o não preenchimento das DOs. A recuperação de dados no prontuário da mãe foi fundamental ao resgate de informações preciosa, as quais os médicos pediatras não tiveram acesso. Essas informações seriam decisivas na condução clínica dos casos, demonstrando falta de diálogo e entrosamento entre as equipes, fundamentais à boa prática médica.

A inconsistência e divergência de dados de mortalidade a partir de estimativas e da multiplicidade de fontes de informações foram questionadas pelo setor saúde em Alagoas ${ }^{31}$. Na ocasião, as autoridades apontaram como fonte de melhor qualidade o Sistema de Informações de Atenção Básica (SIAB) para Alagoas. De acordo com Mello Jorge e Gotlieb ${ }^{32}$, em municípios de Sergipe, Tocantins e Ceará, o SIAB foi capaz de evidenciar um número de eventos bem próximo à realidade e superior ao SIM e SINASC. Victora e Tomasi33 observaram que o SIAB estima, atualmente, taxas de mortalidade infantil para Alagoas de 49,3 por mil, enquanto que o SIM/SINASC indica 30,9 por mil, acreditando uma subnotificação do SIM/ SINASC de 42,8\% e 8,7\% para o SIAB, em Alagoas. No entanto, em localidades como Maceió, com apenas 25\% de cobertura pelo Programa de Saúde da Família (PSF), investir na qualidade do SIM e SINASC parece mais lógico, econômico e viável. Independente da qualidade da cobertura destes outros sistemas, eles são fontes que podem complementar as informações dos sistemas SIM/ SINASC. 


\section{ConclusÃo}

A importância da DO como fonte de informações epidemiológicas, norteadora de medidas de atenção à saúde, é indiscutível. Mas este documento só serve a seus propósitos se apresentar validade e confiabilidade de informações, refletindo verdadeiramente o evento ocorrido.

Este estudo, ao analisar DO preenchidas em todos os hospitais que assistem as gestantes e neonatos na capital alagoana, deixa transparecer a baixa qualidade de informações geradas, decorrente do preenchimento inadequado e incompleto dos documentos oficiais. A precariedade no preenchimento da DO foi independente do tipo de assistência médica, se privada ou pública, a ponto de tornar este documento pouco útil aos seus propósitos. Quando a DO e o SIM foram comparados ao padrão-ouro, as variáveis que apresentaram elevada sensibilidade ou taxa de detecção (sexo, idade gestacional) assim se mantiveram, mesmo quando retirado o papel do acaso pela análise estatística do Indicador Kappa. Apesar da imprecisão da forma como a idade gestacional é informada na DO, este documento e o SIM têm capacidade de informar corretamente estas duas variáveis. Dado essencial na DO, como preditor de risco neonatal, o peso ao nascer, mostrou-se com uma diminuição dos valores da sensibilidade na comparação entre o SIM e o prontuário, refletindo um mau preenchimento do SIM a partir da DO

A baixa concordância entre dados de prontuários, DO e SIM permite considerar a qualidade do SIM como inadequada e aponta para a necessidade de torná-lo um sistema efetivo, principalmente por já apresentar uma cobertura de 100\% dos óbitos hospitalares da cidade. É provável que este estudo reflita a realidade de várias capitais brasileiras. Em pleno século XXI, apenas algumas localidades no Sudeste e Sul do país e uma no Centro-Oeste são detentoras de boa qualidade dos demais dados do SIM6,7.

A gravidade da precária situação de um SIS da magnitude do SIM, em uma capital do país, mesmo inserida num contexto de pobreza como o do Nordeste do Brasil, serve de alerta aos gestores e à comunidade científica do país, não apenas da região. Isto é fato não somente pela importância de um SIS de qualidade para o planejamento de ações de saúde, mas pelo investimento contínuo do Ministério da Saúde em garantir sua operacionalização. Todo o esforço e o custo são perdidos quando o médico deixa de fornecer as informações corretas em um documento essencial e de sua inteira responsabilidade, como a DO, ou quando o sistema não é corretamente alimentado.

A divulgação nos serviços dos resultados das análises epidemiológicas, geradas a partir dos dados coletados, poderá estimular a produção de informações confiáveis.

Diante das observações evidenciadas, as autoras sugerem investimento no treinamento dos médicos, residentes e estudantes de Alagoas no preenchimento das DOs, devendo o ensino do preenchimento de documentos oficiais fazer parte do elenco de informações obrigatoriamente repassadas aos alunos durante toda sua formação e não ficar restrito apenas a graduação. Quanto à operacionalização do SIM, é importante que todas as etapas de análise de consistência de seus dados, já previstas pelo próprio sistema, sejam cumpridas com rigor, evitando a inadequação deste importante banco de dados.

\section{Conflito de interesse: não há.}

\section{SUMMARY}

\section{QUALITY OF DATA ON EARLY NEONATAL DEATHS}

OBJECTIVE. To investigate the quality of official neonatal death data in Maceió, Alagoas.

METHOD. A descriptive study was conducted on early neonatal deaths in hospitals between January 1, 2001, and December 31, 2002, to compare data entry in the Death Certificate (DC) and Mortality Information System (MIS) with a standardized form filled out with data of medical files from the mothers and newborn. The frequency with which the following variables failed to be recorded in the DC and SIM was studied: type of death, address, age of mother, gender, birth weight, and delivery type, age at death and gestational age. MIS reliability was verified using simple concordance, sensitivity and Kappa indicator.

RESULTS. MIS recorded 451 deaths, of which 50 were excluded. Mother's age was omitted from MIS in $44.1 \%$ of cases. $85.7 \%$ to $100 \%$ of the variables not filled in for the DC were recovered from the medical files. There was good concordance between DC and medical files for type of delivery, weight and age. Birth weight and age of mother presented the least concordance between medical files and MIS. MIS presented $69.2 \%$ sensitivity for weight and $36.3 \%$ for age of mother, thus demonstrating little capability to correctly supply information to generate perinatal health indicators.

CONCLUSION. Because of incomplete filling out, quality of the $D C$ becomes precarious and makes the MIS inadequate, even though it covers 100\% of neonatal deaths in Maceió. Inefficiency of the system is increased by failure of MIS technicians to correct errors found and input all the information available. [Rev Assoc Med Bras 2007; 53(5): 389-94]

KEY WORDS: Neonatal mortality. Death Certificates. Information systems. Medical records. Mortality records. Validation study.

\section{REFERÊNCIAS}

I. Conselho Federal de Medicina. Resolução CFM n. 1.779/2005. Diário Oficial União, Brasília (DF). 2005;05 dez. Seção I: I21.

2. Laurenti R, Mello-Jorge MHP. O atestado de óbito. São Paulo; 1993. Centro da OMS para a Classificação de Doenças em Português (MS/ USP/OPAS/OMS). (Série Divulgação I).

3. Menezes AMB, Victora CG, Barros FB. Estudo populacional de óbitos perinatais e infantis: metodologia, validade do diagnóstico e subregistro. J Pediatr (Rio de J). 1997;73(6):383-7.

4. Coutinho SB. Mortalidade neonatal em cinco maternidades da cidade do Recife, 1994. Recife; 1996. [Relatório final de pesquisa apresentado ao UNICEF].

5. Vanderlei LC, Arruda BKG, Frias PG, Arruda S. Avaliação da qualidade de preenchimento das declarações de óbito em unidade terciária de atenção à saúde materno-infantil. IESUS. 2002;। I (1):7-|4.

6. Gomes FBC. Sistemas de Informação sobre Mortalidade: considerações sobre a qualidade dos dados. IESUS. 2002; I I (I):5. 
7. Mansano NH. Monitoramento da mortalidade infantil no Brasil pelos Sistemas de Informação de Mortalidade (SIM) e nascidos vivos (SINASC). In: $6^{\circ}$ Congresso Brasileiro de Epidemiologia, Comunicação Coordenada, Recife; 2004

8. DATASUS. Indicadores de Mortalidade 2004. Número de óbitos infantis (menores de I ano) por I.000 nascidos vivos, Brasil, 19972002. [citado 13 dez 2003]. Disponível em: http:// tabnet.datasus.gov.br/cgi/idb2004/c0 l.htm.

9. Pereira MG. Epidemiologia: teoria e prática._Rio de Janeiro: Guanabara Koogan; 2000.

10. Organização Pan-americana de Saúde. Fundação Nacional de Saúde. Métodos de investigação epidemiológica em doenças transmissíveis. Brasilia (DF); 1997. v.l.

I I . Turrini RNT, Santo AH. Infecção hospitalar e causas múltiplas de morte. J Pediatr (Rio de J). 2002; 78(6):485-90.

12. Barros F, Diaz-Rossello JL. Redes multicêntricas e a qualidade da atenção neonatal. J Pediatr (Rio de J). 2004;80(4):254-6.

13. Santa Helena ET, Rosa MB. Avaliação da qualidade das informações relativas aos óbitos em menores de um ano em Blumenau, 1998. Rev Bras Saude Matern.Infant. 2003;3(I):75-83.

I 4. Mahapatra P, Chalapati RPV. Cause of death reporting systems in India: a performance analysis. Natl Med I India. 200 I:14(3): I54-62.

1 5. Pereira MG, Castro ES. Avaliação do preenchimento de declarações de óbitos: Brasília, DF (Brasil), 1977-1978. Rev Saúde Pública. 198|; | 5(I): | 4-9.

16. Heckmann IC, Sant'anna UL, Bordin R. Análise do preenchimento de declarações de óbitos em localidade do estado do Rio Grande do Sul (Brasil), 1987. Rev Saúde Pública. 1989:23(2):292-7.

17. Gomes JO, Santo AH. MI em Município da Região Centro-Oeste Paulista, Brasil, 1990 a 1992. Rev Saúde Pública. 1997;31(4):33041 .

1 8. Niobey FML, Cascão AM, Duchiade MP, Sabroza PC. Qualidade do preenchimento de atestados de óbito de menores de um ano na Região Metropolitana do Rio de Janeiro. Rev Saúde Pública. 1990;24(4):311-8

19. Freitas MS. Avaliação do Sistema de Informação de Mortalidade no Estado do Acre, no ano de 1994 [dissertação]. Faculdade de Saúde Pública, Universidade de São Paulo; 1997.

20. Vanderlei LC, Arruda BKG, Frias PG, Arruda S. Avaliação da causa básica de óbito em unidade terciária de atenção à saúde maternoinfantil. IESUS. 2002; I I ( I): I5-23.

21 . Swift B, West K. Death Certification: an audit of practice entering the 21 ${ }^{\text {st }}$ century. J Clin Pathol. 2002;55(4):275-9.

22. Morlon L, Omar R, Carrol S. Incomplete and inaccurate death certification: the impact on research. I Public Health Med. 2000;22(2): 133-7.
23. Johansson LA, Westling R. Comparinh Swedish hospital discharge records with death certificates; implications for mortality statistcs. Int | Epidemiol. 2000;29(3):495-502.

24. Hunt R, Barr P. Errors in the certification of neonatal death. J Paediatr Child Health. 200I;36(5):498-50I.

25. Lahti RA, Penttilä A. The validity of death certificates: routine validation of death certification and its effects on mortality statistics. Forensic Sci Int. 200 I; | I5(I-2): I5-32.

26. Lomuto C. De que se mueren los recien nacidos em la Argentina 1995? Rev. Hosp. Mat Inf Ramón Sardá 1998;17(1):31-5.

27. Hertoghe L, De Wals P, Piron M, Bertrand F, Lechat MF. Quality of perinatal death registration. A study in Hainaut, Belgium. Eur J Pediatr. 1987; | |46(5):473-6.

28. Brasil. IBGE. Censo 2000. [citado I3 dez 200 I]. Disponível em: http:/ /www.ibge.gov.br/.

29. Serafim D. Mortalidade neonatal em Maringá-PR, 1997-2000 [tese]. São Paulo: Faculdade de Saúde Pública, Universidade de São Paulo; 2002..

30. Campos MR, Leal MC, Souza Jr PR, Cunha CB. Consistência entre fontes de dados e confiabilidade interobservador do Estudo da Morbimortalidade e atenção Peri e Neonatal no Município do Rio de Janeiro. Cad Saúde Pública. 2004;20(Suppl I):534-43.

31. Machado AA. Mortalidade infantil: a divergência de dados. [citado I4 maio 2003]. .Disponível em http://www.saude.al.gov.br.

32. Mello-Jorge MHP, Gotlieb SLD. O Sistema de Informação de Atenção Básica como Fonte de dados para os sistemas de informações sobre mortalidade e sobre nascidos vivos. IESUS. 200 I;I0(I):7-18.

33. Victora CG, Tomasi E. Análise de tendências temporais na mortalidade infantil de Alagoas, 1999-2002. Pelotas; 2004. [Relatório Final para - UNICEF - Brasil].

Artigo recebido: 14/6/06

Aceito para publicação: 3/8/07 